\title{
Full Mouth Rehabilitation: A Case Report Utilizing Fixed and Removable
}

\author{
Dr.Dinesh Chavan MDS ${ }^{1}$, Dr.Roshani Chavan MDS $^{2}$, \\ Dr.Abhijeet Pharande MDS ${ }^{3}$, Dr.Manish Pharande MDS ${ }^{4}$
}

\section{Introduction}

Dentistry certainly must be an important part of an overall cosmetic upgrade, as improvements in the face and the smile plays an important role in changing a person's appearance and improving self-esteem. The phrase full-mouthrehabilitation means different things to different people. Though the full-mouthrehabilitation and its philosophies are often intrigued in nature, but the esthetic andfunctional accomplishment of rehabilitation is always satisfying.

\section{Case Report}

A healthy 37-year-old female referred from the department of endodontics forrestoration of endodontically treated teeth to the Department of Prosthodontics, atDr.D.Y. Patil Dental College Pimperi, Pune. Patient complains of fractured teeth,difficulty in chewing, unsatisfied esthetic so patient wants to get her faulty anterior

crowns replaced, improve her masticatory efficiency and esthetics. The patient alsogave a dental history that she had undergone orthodontic treatment at the age of 23and for that she had undergone orthodontic extraction of 14 $\& 24$.

\section{Clinical findings}

Extra oral: The patient had no facial asymmetry, or muscle tenderness. Themandibular range of motion was within normal limits. The TMJ, the muscles ofmastication, and the facial expression were asymptomatic.(fig:- 1)

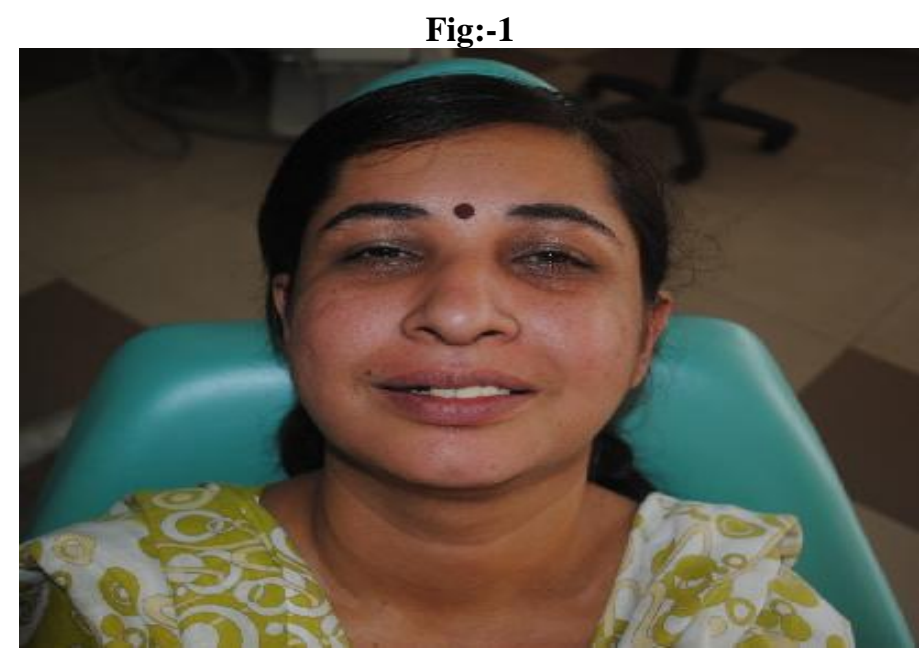

Intraoral:

No gross abnormalities were noted in the overall soft tissues of the lips, cheeks,tongue, oral mucosa, and pharynx. A generalized pitting defect was notedMaxillary arch: faulty crown present with 11, 21, 22, 23 \#13, \#15; missing 12, 24, 25(fig:-2)

Mandibular arch: missing 36, 44, 45, 46 (fig :-3) 


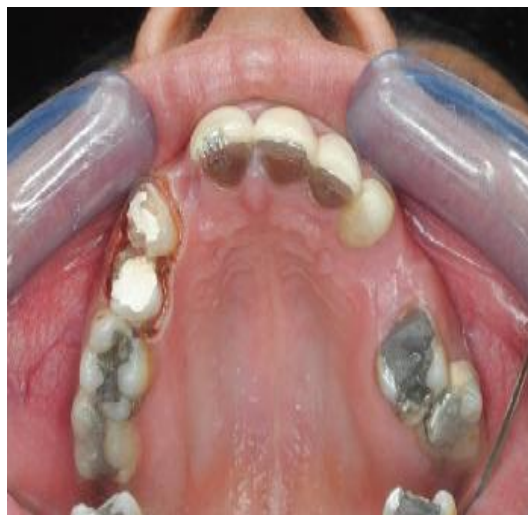

(fig:-2)

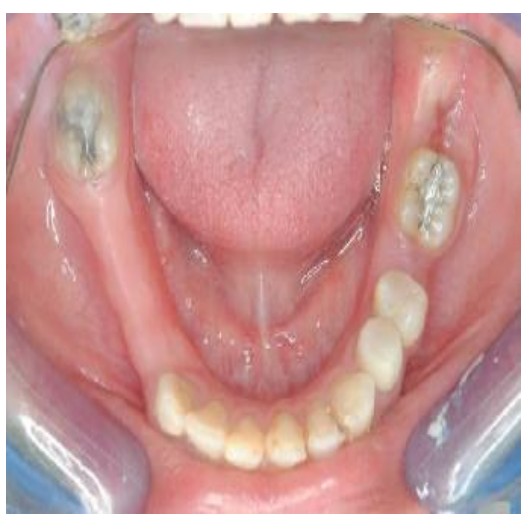

(fig :-3)

\section{Conclusion}

Class I canine relationship \& canine guided the existing occlusion on lateral excretion. Only canine are in contact and posteriors are disoccluded.

\section{Goals of rehabilitation :-}

The goals are as follows:

1) Correction of occlusal plane

2) Masticatory efficiency

3) Improvement in esthetics

\section{Treatment planning preprosthetic phase:}

- Oral prophylaxis

- $\quad$ Extraction with 18, 12, 28, 38

- $\quad$ Root canal with 26,27

\section{Prosthetic phase:}

- Tooth preparation

- Temporization

- Final impression

- Cementation of final restoration

\section{Maintenance phase:}

- Evaluation of patient comfort

- Evaluation of prosthesis

- Evaluation of oral hygiene

Treatment plan is executed as follows:

I] preprosthetic phase:

Oral prophylaxis: scaling and root planning.

Extraction with 18, 12, 15, 28, 38

Root canal with 26, 27

\section{II] prosthetic phase:}

1) Maxillary and mandibular impressions were made in the alginate impressionmaterial and diagnostic casts were obtained.

2) Then the maxillary cast was mounted using face-bow transfer (ear piecetype) onto a semi-adjustable articulator (Hananu H2) and the mandibular cast was mounted by using centric record established by bilateral manipulation method. 


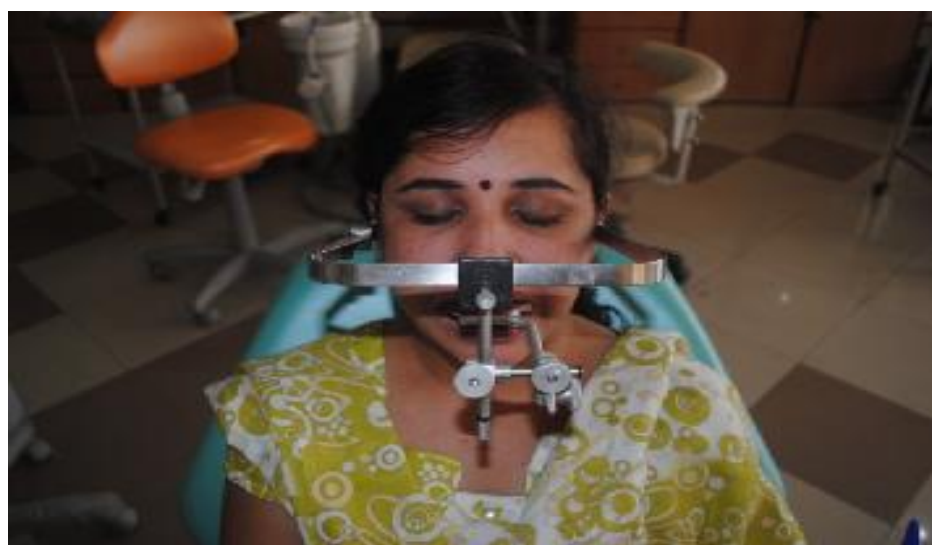

Fig:-4

3) Mounted U/L diagnostic cast

4) Protrusive record was made to determine horizontal condyler guidance. Byusing this the lateral condylar guidance was determine by the Hanau'sformula $(\mathrm{L}=\mathrm{H} / 8+12)$

5) Anterior wax up was done.

6) To establish the mandibular occlusal plane Broadricks plane analysis was done.(broadrick plane analysis) (fig

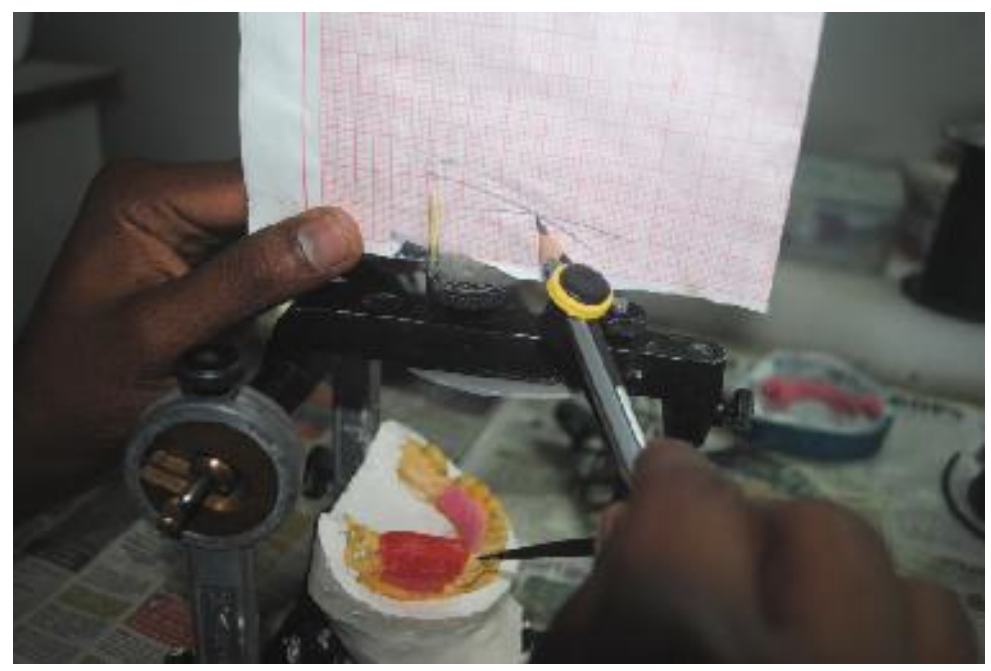

fig :-5

7) According to the analysis the mandibular wax up is done. Following this themaxillary wax up is also completed.( Fig :-6)

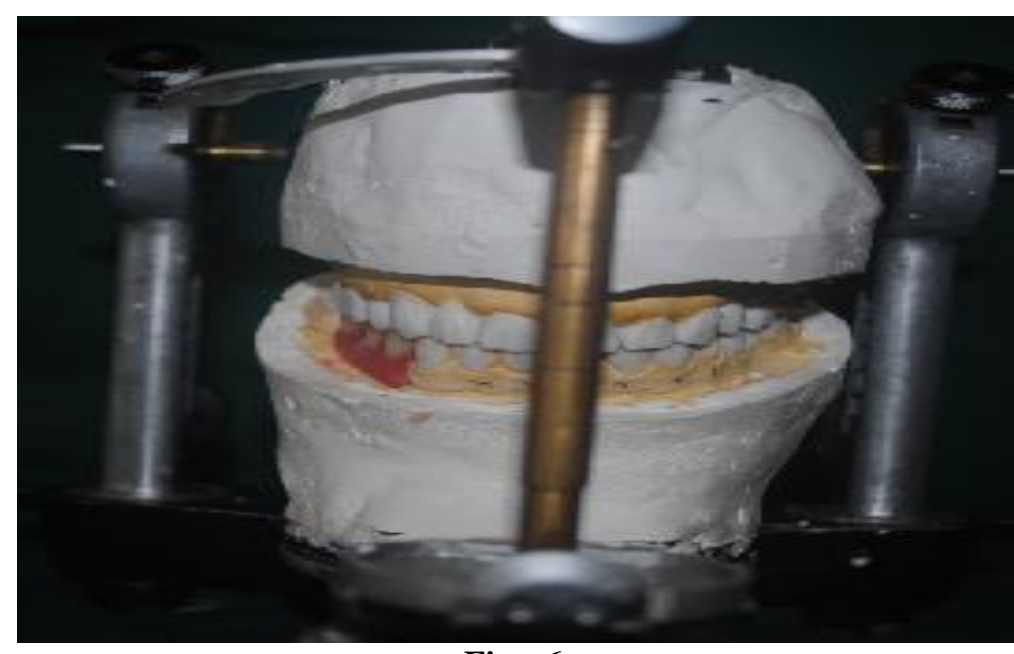

Fig :-6 
8) Tooth preparation was carried out quadrant wise (Sequence is as follows: 1st and 4th quadrant, followed by temporization to establish vertical dimension of occlusion. Then the2nd and 3rd quadrant followed by temporization)(fig :-7)

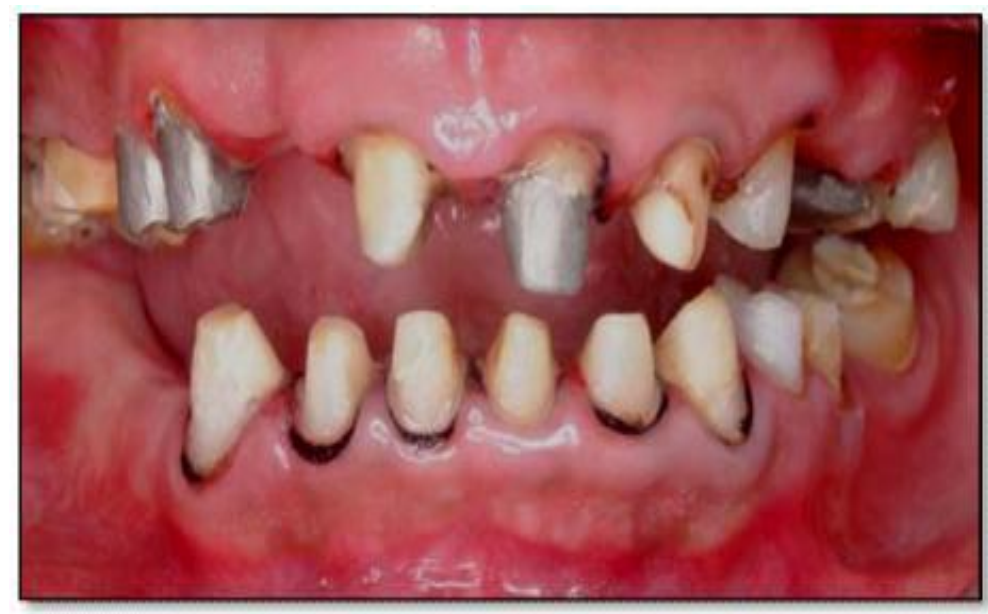

fig :-7

9) Temporization is kept for 3 weeks and patient comfort was evaluated by phonetics and esthetics. (fig :- 8)

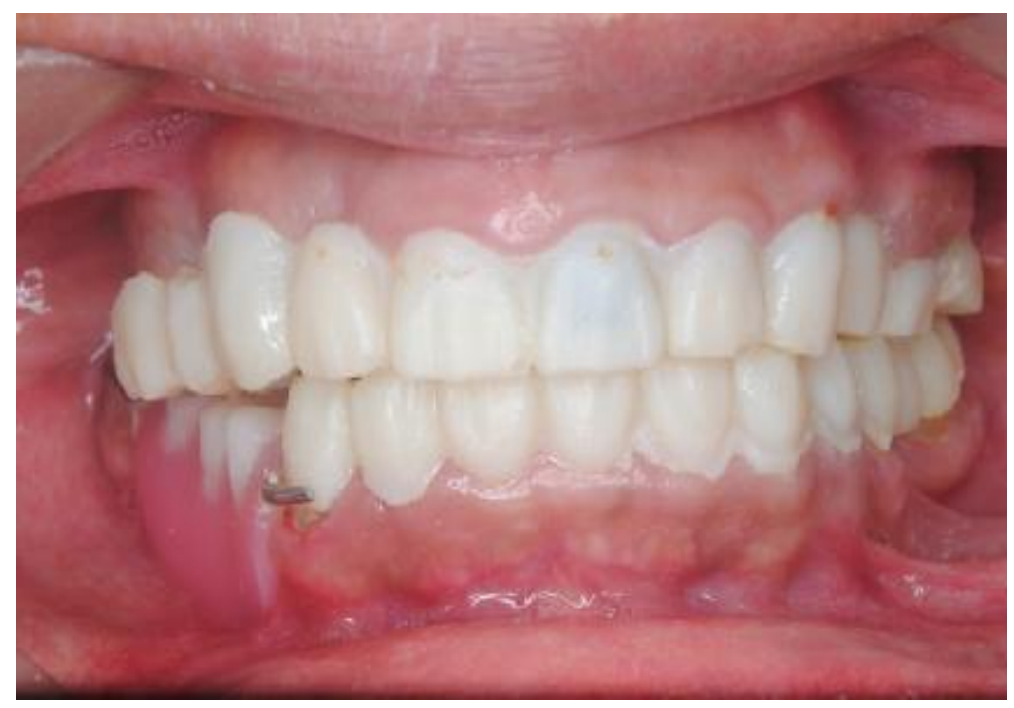

fig :- 8

10) Temporization was removed and gingival retraction cord was placed.

11) Final impressions were made with the help of light body putty (3MESPE) single step method.

12) Master cast was made.

13) Face bow record was taken for mounting the maxillary master cast.For mounting the mandibular master cast lucia jig was fabricated andcentric record was made by bilateral manipulation method.(lucia jig)

14) For maintaining the same vertical as in the temporization, anteriortemporization is removed and posterior temporization was placed in the patient's mouth, low fusing compound was placed on the anterior teeth and patient was asked to bite on it in centric relation.

15) Posterior temporizes were removed and wax sheet was used to record the centric relation. On this zinc oxide eugenol was placed to preserve the accuracy of the record.

16) Lower master cast was mounted.

17) Coping was fabricated on this and coping trial was done.

18) Bisque trial was done.

19) Final restoration was placed. (Fig :-9) 


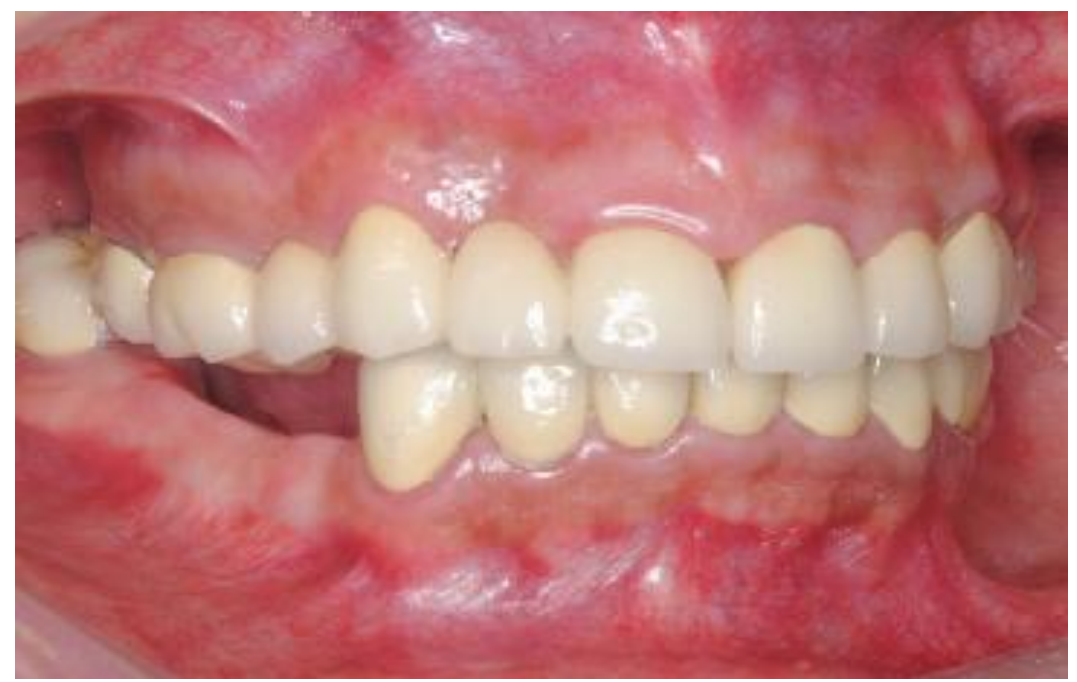

Fig :-9

20) For fabrication of cast partial framework the rest were preplan in thefinal cemented crown on $35,36,43$, and 47.

21) Final impression was made in light body putty (3M ESPE) for fabrication of cast partial frame work.

22) Frame work was checked and jaw relation was made.

23) Try in done.

24) Final prosthesis insertion done. (Fig :-10)

Fig :-10

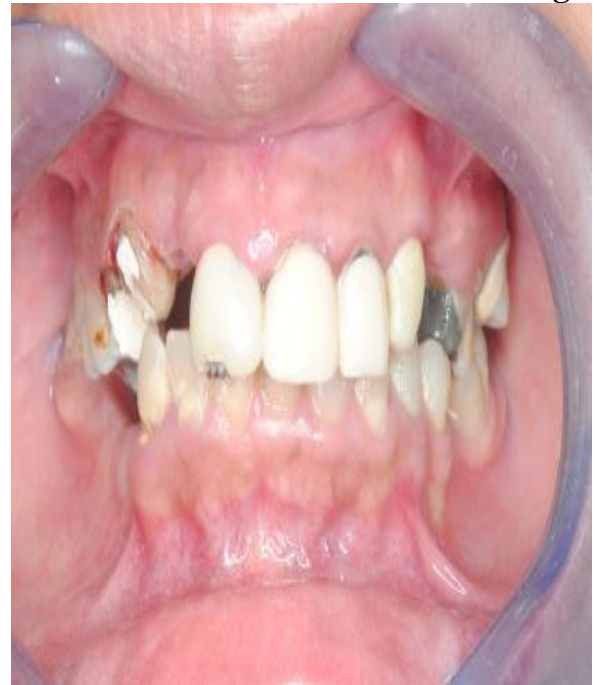

Pre-op photograph

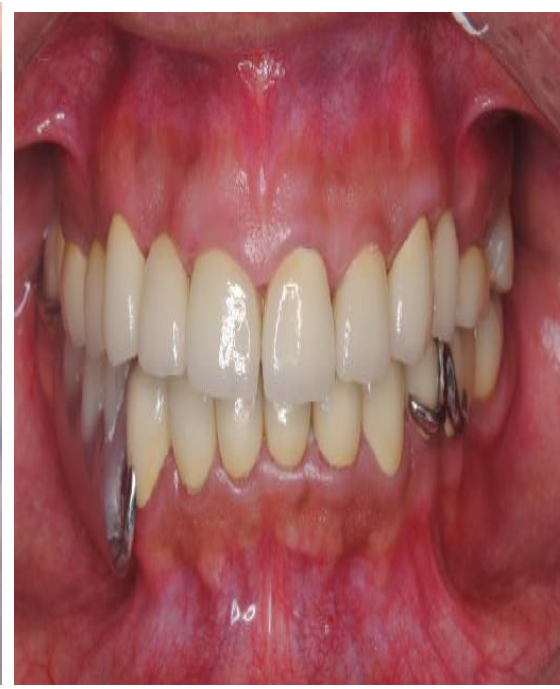

Post-op photograph 